\title{
Novel Layered Double Hydroxides@Carboxymethyl Cellulose Composite Aerogel Towards Co(II) Absorption
}

Wang Liao ( $\sim$ liaowang0624@126.com )

Xihua University https://orcid.org/0000-0002-8588-3492

\section{Xinlei Long}

Xihua University

\section{Yuping Wei}

Xihua University

Yao Xiao

Xihua University

\section{Research Article}

Keywords: Layered double hydroxides, carboxymethyl cellulose, composite aerogel, absorption, cobalt ion.

Posted Date: January 17th, 2022

DOI: https://doi.org/10.21203/rs.3.rs-1236917/v1

License: (c) (i) This work is licensed under a Creative Commons Attribution 4.0 International License. Read Full License 


\title{
Novel layered double hydroxides@ carboxymethyl cellulose composite aerogel towards Co(II) absorption
}

\author{
Wang Liao ${ }^{*}$, Xinlei Long, Yuping Wei, Yao Xiao \\ School of Science, Xihua University, Chengdu 610039, Sichuan, China. \\ Email: liaowang@mail.xhu.edu.cn (W. Liao), ORCID: 0000-0002-8588-3492 (W. Liao)
}

\begin{abstract}
Cobalt $(\mathrm{Co})$ ion is a common heavy metal ion and the main metal ion in nuclear wastewater. However, the study on the absorption for Co(II) is not sufficient. Herein, a novel ES-layered double hydroxide (LDH) is prepared by intercalated ethylenediaminetetraacetic acid disodium salt (EDTA) and sodium dodecylbenzene sulfonate (SDBS) in a magnesium, iron pristine LDH, which is subsequently used for ES-LDH@carboxymethyl cellulose (CMC) composite aerogel. The results of Fourier transform infrared spectroscopy, X-ray diffraction spectra and thermogravimetric analysis shows successfully preparation of intercalated LDH. The pore morphology, specific surface area and pore size distribution of the composite aerogels are characterized by scanning electron microscope and Nitrogen-BET curves. The significantly improved adsorption capacity, adsorption kinetics and isotherms the absorbents on Co(II) are systematically revealed. This study thus provides an important understanding and path for dealing with the threat of $\mathrm{Co}(\mathrm{II})$.
\end{abstract}

\section{Keywords}

Layered double hydroxides; carboxymethyl cellulose; composite aerogel; absorption; cobalt ion.

\section{Introduction}

With the global efforts towards Carbon Neutrality, it will be a developing trend to promote application of clean energy, including safe and stable use of nuclear energy. The isotope of Cobalt (Co), i.e. ${ }^{60} \mathrm{Co}$, are one of the main pollution sources of nuclear wastewater. Furthermore, Co is a typical heavy metals with density greater than $4.5 \mathrm{~g} / \mathrm{cm}^{3}$. Heavy metals are widely used in the manufacture of electronic components, antibacterial materials, batteries and photosensitive materials. Attention must be paid to the post-treatment of the heavy metal ions (HMIs). In case of improper treatment, HMIs will cause serious environmental and health crisis. Excessive HMIs in a body can lead to a variety of diseases, and 
the limit is actually extremely low $[1,2]$. The main reason is that HMIs can inactivate human protein, and the organism can not metabolize HMIs, which aggravate through the food chain [3]. Various methods have been applied to eliminate HMIs pollution, such as precipitation, electrolysis, membrane separation, ion exchange and adsorption [4-7]. Among them, adsorption is a common phenomenon at the solid-liquid interface. The adsorption and subsequent desorption of adsorbate have the advantages of high efficiency, fast speed, low cost, simple operation and no secondary pollution. In addition, the adsorbent has the advantages of reusability, which is therefore widely concerned and applied $[5,8,9]$. Nevertheless, the study on the adsorption of cobalt ions is not sufficient.

Layered double hydroxides (LDHs) are layered clay compounds with positively charged hydroxide layers and interlayer anions. The general chemical formula of LDHs is: $\left[\mathrm{M}(\mathrm{II})_{1-\mathrm{X}} \mathrm{M}(\mathrm{III})_{\mathrm{X}}(\mathrm{OH})_{2}\right]^{\mathrm{X}+}\left[\mathrm{A}^{\mathrm{n}-}{ }_{\mathrm{x} / \mathrm{n}}\right] \cdot \mathrm{mH}_{2} \mathrm{O}$ (where $\mathrm{M}^{2+}$ and $\mathrm{M}^{3+}$ are divalent and trivalent metal cations, $\mathrm{A}^{\mathrm{n}-}$ is interlayer anion, and $\mathrm{X}=\mathrm{M}^{3+} /\left(\mathrm{M}^{2+}+\mathrm{M}^{3+}\right)$, with a value of $\left.0.2-0.34\right)[10,11]$. The composition can be adjusted in a certain range, and the layered structure has a large specific surface area, which makes them have high adsorption performance in a variety of adsorption substrates [12, 13]. However, due to the lack of specific function and structure, the adsorption performance of pristine LDH is limited [14]. In addition, it is critical to fabricate high performance materials by compounding other components with functionalities, pristine LDH can hardly meet the requirements. For instance, a bio-diprotic acid intercalated LDH and polyvinyl chloride composites [15] and humic acid and LDH composites [16] for cadmium ion removal. Composite materials of nano-iron(0) fixed LDH [17], ZIF-8 fixed CoAl-LDH carbon fiber [18], electrospinning fiber membrane with EDTA intercalated LDH [19], and silanized citric acid intercalated LDH-polyaniline [20] have excellent adsorption and separation of a variety of HMIs. Nevertheless, there are still some points should be noted, such as high cost of raw materials, secondary pollution in post-treatment and complicated separation process, etc. Therefore, it is of great value to prepare LDH based adsorption materials with no secondary pollution and easy separation from waste water by a simple method.

Carboxymethyl cellulose (CMC) is a widely used modified anionic polysaccharide with biocompatibility and biodegradability, which can be obtained by one-step reaction from cellulose [14, $18,19]$. In recent years, the CMC based hydrogels develops rapidly [12, 13, 17]. These researches prove that $\mathrm{CMC}$ is a competent component for HMIs removal. In this work, a novel intercalated magnesium $(\mathrm{Mg}$ ) and iron ( $\mathrm{Fe}$ )-based LDH is prepared, which is fabricated with CMC to obtain a 
composite aerogel. This aerogel is tested as an adsorbent to remove $\mathrm{Co}(\mathrm{II})$, and the adsorption behaviors and mechanism are revealed. It is believed that this study provides an important basis for dealing with threat of $\mathrm{Co}(\mathrm{II})$ and design of novel absorbents.

\section{Experimental section}

\subsection{Materials}

$\mathrm{FeCl}_{3} \cdot 6 \mathrm{H}_{2} \mathrm{O}, \mathrm{MgCl}_{2} \cdot 6 \mathrm{H}_{2} \mathrm{O}, \mathrm{NaOH}, \mathrm{Na}_{2} \mathrm{CO}_{3}$, cobalt(II) acetate [Co(Ac) $)_{2}$, ethylenediaminetetraacetic acid disodium salt (EDTA), sodium dodecylbenzene sulfonate (SDBS), sodium carboxymethylcellulose (CMC) and glutaraldehyde aqueous solution (50\%) are analytical reagents which are obtained from Chengdu Kelong Chemicals Co., Ltd. Deionized (DI) water (resistivity $>2.0 \mathrm{~m} \Omega \cdot \mathrm{cm}$ ) is used in all involved experiments.

\subsection{Preparation of LDHs}

2.2.1 Prepartion of pristine $\mathrm{MgFe}-\mathrm{LDH} . \mathrm{Na}_{2} \mathrm{CO}_{3}(0.06 \mathrm{~mol})$ and $\mathrm{NaOH}(0.1 \mathrm{~mol})$ were dissolved in a $500 \mathrm{~mL}$ three-necked flask with $100 \mathrm{~mL}$ water and heated in a $80^{\circ} \mathrm{C}$ oil bath. $\mathrm{FeCl}_{3} \cdot 6 \mathrm{H}_{2} \mathrm{O}(0.06 \mathrm{~mol})$ and $\mathrm{MgCl}_{2} \cdot 6 \mathrm{H}_{2} \mathrm{O}(0.02 \mathrm{~mol})$ were dissolved in $100 \mathrm{~mL}$ water and then transferred to a constant pressure dropping funnel. This solution was slowly dropped into the flask and the $\mathrm{pH}$ value was controlled at about 10. After a reaction of $30 \mathrm{~min}$, the solution was transferred to a beaker and aged in a oven at $65^{\circ} \mathrm{C}$ for $24 \mathrm{~h}$. The product was centrifuged at low-speed, washed and freeze-dried to obtain pristine $\mathrm{Mg}(\mathrm{II}), \mathrm{Fe}(\mathrm{III})-\mathrm{CO}_{3}{ }^{2-}-\mathrm{LDH}(a b b r . \mathrm{MgFe}-\mathrm{LDH})$.

2.2.2 Preparation of $E S-L D H$. The pristine $\mathrm{MgFe}-\mathrm{LDH}$ powder was calcined at $500^{\circ} \mathrm{C}$ in muffle furnace for $4 \mathrm{~h}$. During this process, the interlayer anions, bound water and some hydroxyls were removed to obtain double oxide (LDO). The LDO was put in $200 \mathrm{~mL}$ solution of 0.02 mol EDTA and/or 0.02 mol SDBS, and stirred for $24 \mathrm{~h}$. Again, the product was centrifuged, washed and freeze-dried to obtain EDTA and SDBS intercalated MgFe-EDTA-LDH, MgFe-SDBS-LDH and MgFe-LDH (ES-LDH) (Scheme 1). 


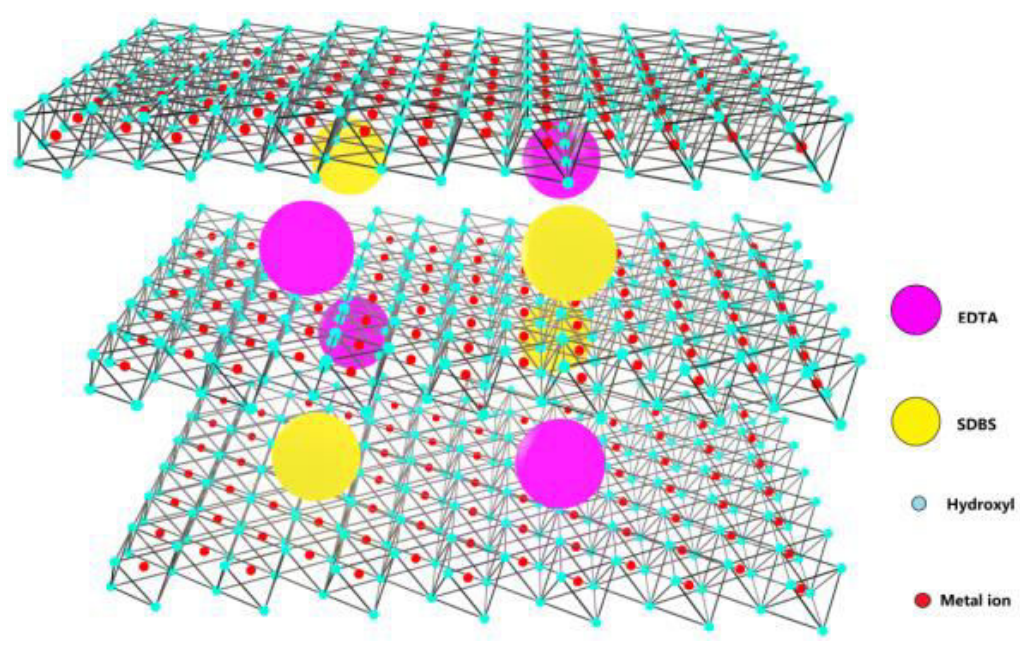

Scheme 1. Structure of ES-LDH.

\subsection{Preparation of ES-LDH@CMC composite aerogel}

ES-LDH (0.5 g) was first dispersed in $95 \mathrm{~mL}$ water, after which CMC (4.5 g) was added in it until the system completely swelled to a homogeneous jelly-like intermediate product. After that, sulfuric acid was added to make the $\mathrm{pH}$ value about 6 . Then $10 \mathrm{~mL}$ glutaraldehyde (aq) was added into it for crosslinking $24 \mathrm{~h}$. The obtained product was ES-LDH@CMC composite hydrogel. Unreacted small molecules were removed by dialysis. The hydrogel was further freeze casted and freeze dried to obtain ES-LDH@CMC-10 composite aerogel, in which the number '10' indicates $10 \%$ of ES-LDH in dry matter.

\subsection{Absorption experiments of $\mathrm{Co}$ (II)}

Composite aerogel sample $(0.1 \mathrm{~g})$ was put in a gauze and immersed in the $\mathrm{Co}(\mathrm{Ac})_{2}(100 \mathrm{mg} / \mathrm{L})$ solution for some time. After the absorption, the liquid was taken out and centrifuged at $10000 \mathrm{rpm}$ to obtain the supernatant, which was diluted 4 times. The solution was put in a polytetrafluoroethylene beaker and added aqua regia $(4 \mathrm{~mL})$ and hydrofluoric acid $(0.05 \mathrm{~mL})$. This solution was left for $15 \mathrm{~min}$ at RT and then heated to $150^{\circ} \mathrm{C}$ for digesting organic molecules on until the solution was clear. After cooling down, the solution was diluted in a $10 \mathrm{~mL}$-volumetric flask. The concentration of the rest $\mathrm{Co}(\mathrm{II})$ was determined by ICP-OES.

According to the same method, kinetic analysis was carried out at $\mathrm{T}=20^{\circ} \mathrm{C}$ and $\mathrm{pH}=7.0$, solution sample was taken out every $1 \mathrm{~h}$ to determine the adsorption capacity. Furthermore, $\operatorname{Co}(\mathrm{Ac})_{2}$ solutions with different concentrations of 50, 65, 80, 105, 150 and $200 \mathrm{mg} / \mathrm{L}$ were prepared and the isotherms under the condition of $\mathrm{T}=20^{\circ} \mathrm{C}, \mathrm{pH}=7.0$ and adsorption time of $6 \mathrm{~h}$ were recorded. 


\subsection{Absorption kinetics and isotherms}

The calculation method of adsorption capacity $\left(q_{e}, \mathrm{mg} / \mathrm{g}\right)$ at some time is:

$$
q_{e}=\frac{c_{0}-c_{t}}{m} V
$$

where $c_{0}$ is the initial $\mathrm{Co}(\mathrm{II})$ concentration $(\mathrm{mg} / \mathrm{L}), c_{t}$ is the $\mathrm{Co}(\mathrm{II})$ concentration at the tested time, $V$ is the volume of the suspension (L), and $m$ is the mass of the adsorbent $(\mathrm{g})$. The adsorption kinetics include [21-24]

(1) Pseudo-first-order kinetic. The integrated rate law form of the pseudo-first-order equation is described by Eq. (2):

$$
\lg \left(q_{e}-q_{t}\right)=\lg q_{e}-\frac{k_{1}}{2.303} t
$$

in which the deduced coefficient $k_{l}$ is considered as an indicator for mass transfer coefficient. The value $q_{t}$ is the adsorption capability at time $t$.

(2) Pseudo-second-order kinetic. This model has been widely applied to a number of metal/absorbent systems. The integrated form of the second-order kinetics equation is described in the following form:

$$
\frac{t}{q_{t}}=\frac{1}{k_{2} q_{e}^{2}}+\frac{t}{q_{e}}
$$

(3) Intra-particle diffusion equation. Intra-particle diffusion model is commonly used for discussing the adsorption mechanism. According to this model, for most adsorption processes, the uptake varies almost proportionately with $t^{0.5}$ rather than with the contact time:

$$
q_{t}=k_{i} t^{0.5}+C
$$

where $t^{0.5}$ is the square root of the time and $k_{i}\left[\mathrm{mg} /\left(\mathrm{g} \cdot \mathrm{min}^{0.5}\right)\right]$ is the rate constant.

(4) Elovich equation. Elovich equation is commonly expressed as Eq. (5):

$$
q_{t}=\frac{1}{\beta} \ln (\alpha \beta)+\frac{1}{\beta} \ln t(5)
$$

in which $\alpha[\mathrm{mg} /(\mathrm{g} \cdot \mathrm{min})]$ is the initial adsorption rate and $\beta(\mathrm{g} / \mathrm{mg})$ is the desorption constant related to the extent of the surface coverage and activation energy for chemical absorption. Elovich equation could confirm a chemisorption. However, it could not predict any definite mechanism.

The adsorption equilibrium is usually described by an isotherm equation, in which the parameters express the surface property of the absorbent and affinity between the absorbent and the absorbate [25-27]. For the newly developed composite aerogels in this study, different isotherm models are 
essentially required to discuss the absorbing behavior, which include

(1) Langmuir isotherm. This model has been widely applied to many metal ions absorption process, which takes the following form:

$$
\frac{c_{e}}{q_{e}}=\frac{1}{q_{m} k_{L}}+\frac{c_{e}}{q_{m}}(6)
$$

where $q_{m}$ is the quantity of adsorbate required to form a single monolayer on unit mass of adsorbent (mg/g). $q_{e}$ is the amount adsorbed on unit mass of the adsorbent $(\mathrm{mg} / \mathrm{g})$ when the equilibrium concentration is $c_{e}(\mathrm{mg} / \mathrm{L}) . k_{L}(\mathrm{~L} / \mathrm{mg})$ is Langmuir constant that is related to the apparent energy of adsorption.

(2) Freundlich isotherm. This model relates the concentration of an absorbate on the surface of an adsorbent. The heat of adsorption decreases in magnitude with increasing the extent of adsorption. This isotherm is commonly expressed as:

$$
\ln q_{e}=\frac{1}{n} \frac{1}{\ln c_{e}}+\ln k_{F}(7)
$$

where $k_{F}\left[\left(\mathrm{mg}^{1-1 / n} \mathrm{~L}^{1 / \mathrm{n}}\right) / \mathrm{g}\right]$ and $n(\mathrm{~g} / \mathrm{L})$ are the deduced constants of the system, indicating the relative adsorption capacity of the adsorbent related to the bonding energy and the adsorption intensity, respectively.

(3) Tempkin isotherm. In this mode, the uniformly distributed adsorbates repulse each other, and the heat of adsorption decreases linearly. This equation is given by Eq. (8):

$$
q_{e}=B_{T} \ln A_{T}+B_{T} \ln c_{e}
$$

where $B_{T}=R T / b_{T}, T(\mathrm{~K})$ is the absolute temperature and $R[8.314 \mathrm{~J} /(\mathrm{mol} \cdot \mathrm{K})]$ is the universal gas constant. The constant $b_{T}$ is related to the heat of adsorption, $A_{T}$ is the equilibrium binding constant (L/min) corresponding to the maximum binding energy.

(4) Dubinin-Radushkevich $(D-R)$ isotherm. This model is deduced from the mean free energy of adsorption $(E)$. If the value of $1 \leq E \leq 16 \mathrm{~kJ} / \mathrm{mol}$, then physical adsorption prevails, and if $E>16$ $\mathrm{kJ} / \mathrm{mol}$, then chemisorption prevails. D-R equation is represented as Eq. (7):

$$
\ln q_{e}=\ln q_{\max }-B \varepsilon^{2}
$$

where $B\left(\mathrm{~mol}^{2} / \mathrm{kJ}^{2}\right)$ is a constant related to mean adsorption energy and $\varepsilon$ is the Polanyi potential, which can be further calculated from Eq. (8):

$$
\varepsilon=R T \ln \left(1+\frac{1}{c_{e}}\right)
$$

The the mean free energy of adsorption can be deduced from: 


$$
E=\frac{1}{\sqrt{-2 B}}
$$

\subsection{Characterization}

The Fourier transform infrared spectroscopy (FTIR) of LDH and ES-LDH were determined by Nicolet 380 spectrometer, Thermo Fisher Scientific, Ltd.

The X-ray diffraction spectra of $\mathrm{Mg}(\mathrm{OH})_{2}, \mathrm{Fe}(\mathrm{OH})_{3}$, LDH and ES-LDH were recorded by Bruker AXS D8 advanced X-ray diffractometer. The testing conditions were tube current $40 \mathrm{~mA}$, tube voltage $40 \mathrm{kV}$, copper test target, diffraction angles were $5-50^{\circ}$ the scanning speed at $10^{\circ} / \mathrm{min}$ and the scanning wavelength at $0.154 \mathrm{~nm}$.

The thermogravimetric curve of ES-EDH was recorded by TGA 550 thermogravimetric analyzer, TA instruments, Ltd. The conditions were $\mathrm{N}_{2}$ atmosphere, gas flow rate of $30 \mathrm{~mL} / \mathrm{min}$ and heating rate of $10^{\circ} \mathrm{C} / \mathrm{min}$

The scanning electron microscope (SEM, Zeiss Merlin Compact) was used for the morphology of the aerogels.

The specific surface area and pore size distribution of the aerogels were characterized by ASAP 2460 $\mathrm{N}_{2}$-BET full automatic surface area and porosity analyzer, Micromeritics, Ltd. The degassing was operated at $80^{\circ} \mathrm{C}$ for $8 \mathrm{~h}$.

\section{Results and discussion}

\subsection{Characterization of ES-LDH}

First, the pristine MgFe-LDH matrix and it derived ES-LDH are analyzed by FTIR. In Figure 1, the large absorption peak near $3400 \mathrm{~cm}^{-1}$ corresponds to the stretching vibration of $-\mathrm{OH}$ of interlayer of LDH plate and interlayer bound water. This wide and large peak not only indicates a lot of hydroxyls, but also corresponds to the association effect of hydrogen bonds. The peak at $1637 \mathrm{~cm}^{-1}$ corresponds to the $\mathrm{C}=\mathrm{O}$ stretching vibration of $\mathrm{CO}_{3}{ }^{2-}$ in $\mathrm{LDH}$ and the carboxycarbonyl stretching vibration of EDTA in ES-LDH [28]. The absorption peak at $500-600 \mathrm{~cm}^{-1}$ is the M-O stretching vibration peak of metal ions coordinated with hydroxyl oxygen in LDH layer [29]. In addition, three peaks between $2800-2980 \mathrm{~cm}^{-1}$ are attributed to the antisymmetric stretching of saturated alkyl groups on SDBS, several small peaks about $1500 \mathrm{~cm}^{-1}$ are attributed to the stretching of SDBS benzene ring skeleton [30]. The peak at 1193 $\mathrm{cm}^{-1}$ corresponds to the stretching vibration peak of $\mathrm{C}-\mathrm{O}$ [31], and the peak at $1132 \mathrm{~cm}^{-1}$ corresponds to 
the stretching vibration peak of $\mathrm{S}=\mathrm{O}$ of SDBS sulfonic groups [30]. Comparing with the absorption spectrum of pristine LDH, all of the mentioned absorption peaks are new for ES-LDH. It can be inferred that EDTA and SDBS are successfully intercalated into LDH.

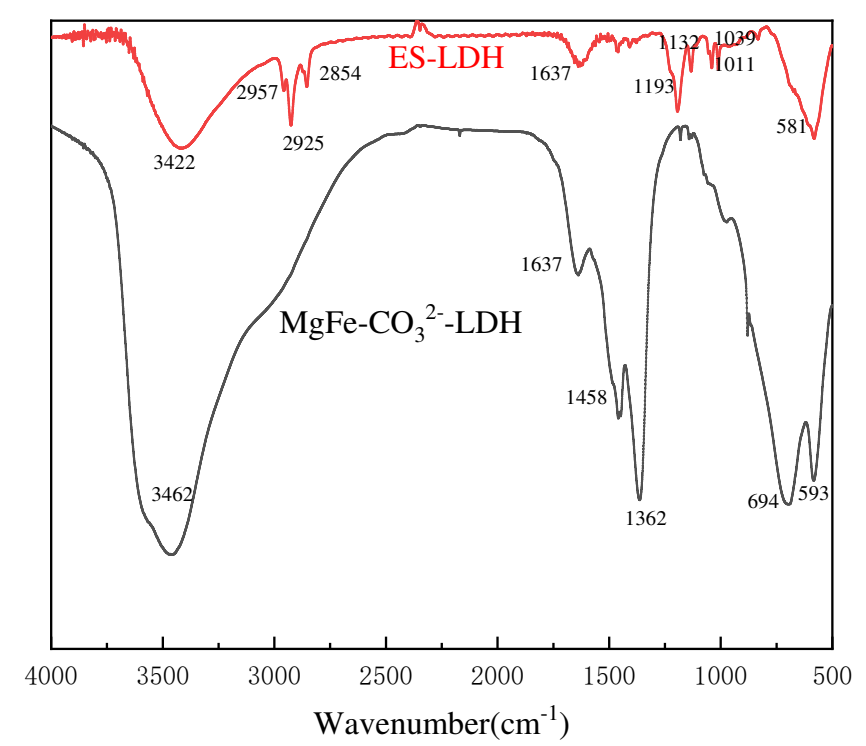

Figure 1. FTIR spectra of pristine LDH and ES-LDH.

Subsequently, the XRD spectra of $\mathrm{Mg}(\mathrm{OH})_{2}, \mathrm{Fe}(\mathrm{OH})_{3}, \mathrm{MgFe}-\mathrm{LDH}$ and ES-LDH are measured and shown in Figure 2. The $2 \theta$ diffraction of crystal faces of MgFe-LDH and ES-LDH are summarized in Table 1. In the XRD spectra, both pristine MgFe-LDH and ES-LDH have the characteristic peaks of (003), (006), (009) and (110) planes. In which, the low values are sharp and strong, while the high values are weak, corresponding to the typical LDH diffraction peaks [32-34]. Comparing Figure 2a and Figure $2 b$, it can be found that the characteristic diffraction peaks of $\mathrm{Mg}(\mathrm{OH})_{2}$ and $\mathrm{Fe}(\mathrm{OH})_{3}$ disappear in the XRD spectrum of ES-LDH. It therefore can be deduced that both MgFe-LDH and ES-LDH are successfully prepared, and the product of ES-LDH is not merely the mixture of $\mathrm{Mg}(\mathrm{OH})_{2}$ and $\mathrm{Fe}(\mathrm{OH})_{3}$. The $2 \theta$ of (003) plane in MgFe-LDH is equal to $11.5^{\circ}$ and $2 \theta$ of (003) plane in ES-LDH is less than $5^{\circ}$. The main reason for this result is that SDBS and EDTA molecules are larger than $\mathrm{CO}_{3}{ }^{2-}$. Once SDBS and EDTA, especially SDBS, enter the interlayers of LDH, the interlayer spacing increases. In addition, there are many burr peaks in the diffraction spectrum of ES-LDH, indicating that the crystallinity of ES-LDH decreases. This phenomenon could be attributed to that the intercalation of SDBS and EDTA increases spacing of the interlayer too much and decreases the crystallinity. 

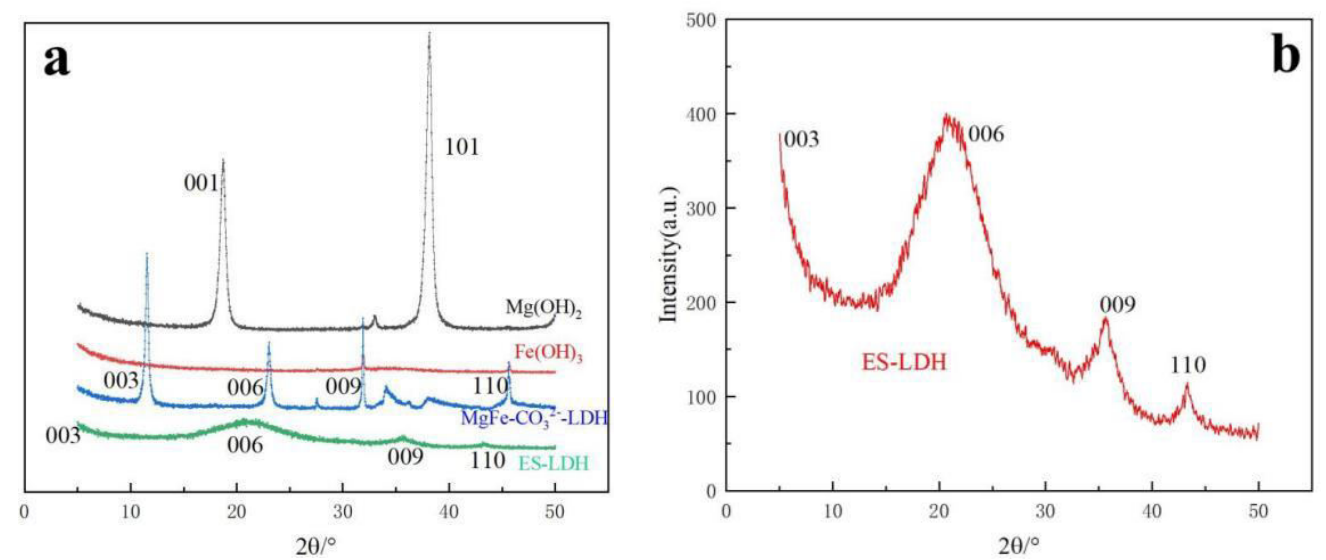

Figure 2. a) XRD spectra of $\mathrm{Mg}(\mathrm{OH})_{2}, \mathrm{Fe}(\mathrm{OH})_{3}, \mathrm{MgFe}-\mathrm{LDH}$ and ES-LDH. b) Enlarged XRD spectrum of ES-LDH.

Table 1. Crystal plane diffraction $2 \theta$ of MgFe-LDH and ES-LDH

\begin{tabular}{ccccc}
\hline \multirow{2}{*}{ Sample } & \multicolumn{5}{c}{ Crystal plane diffraction $2 \theta\left(^{\circ}\right)$} \\
& $(003)$ & $(006)$ & $(009)$ & $(110)$ \\
\hline MgFe-LDH & 11.5 & 23.0 & 31.8 & 45.6 \\
ES-LDH & $<5$ & 20.8 & 35.5 & 43.3 \\
\hline
\end{tabular}

The TG and DTG curves of ES-LDH are shown in Figure 3. From which, ES-LDH begins to decompose at $100^{\circ} \mathrm{C}$ with a weight loss of $2.2 \%$, mainly due to the thermal desorption of adsorbed water between $\mathrm{LDH}$ layers, but the layered structure does not damaged. From 210 to $570^{\circ} \mathrm{C}$, the weight loss is $40.4 \%$, which is the main loss range for ES-LDH. According to the DTG curve, the hydroxyl decomposition dehydration reaction starts at $200-300^{\circ} \mathrm{C}$, with the highest weight loss at $400-450^{\circ} \mathrm{C}$, which corresponds to the decomposition and release of interlayer SDBS and EDTA, collapse the layered structure and formation of LDO [35, 36]. Therefore, these TG-DTG curves are consistent with the typical thermogravimetric curves of LDH. 


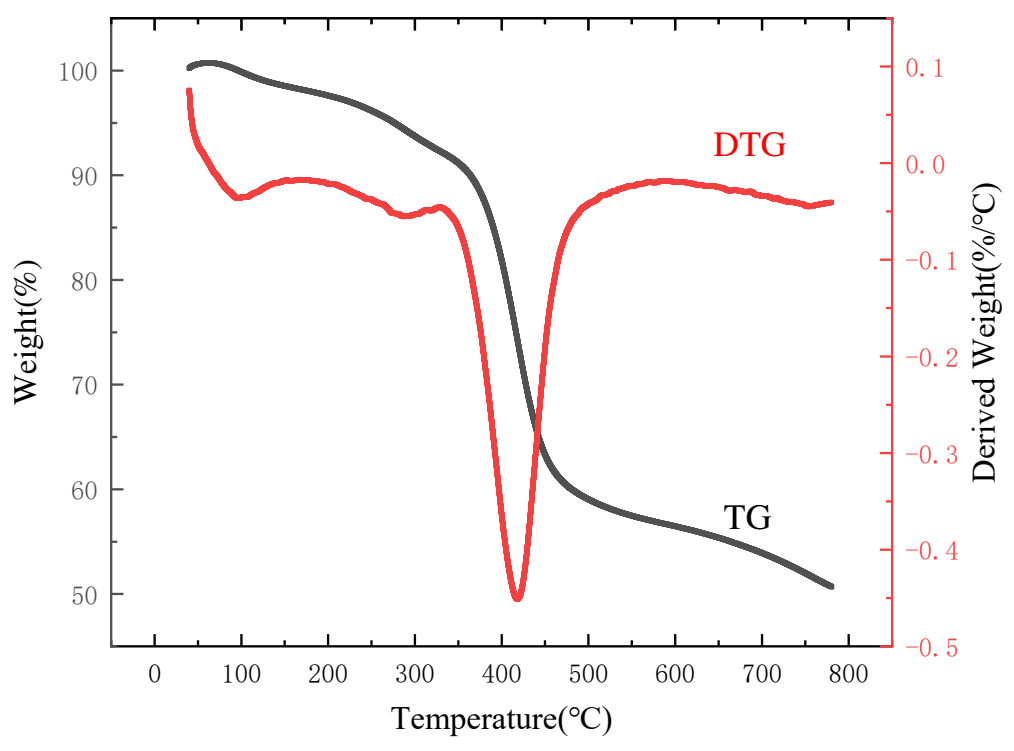

Figure 3. TG and DTG curves of ES-LDH.

\subsection{Characterization of ES-LDH@CMC}

Figure 4 shows the SEM figure of a representative composite aerogel of ES-LDH@CMC-30. From which, it can be clearly seen that the internal micro-structure of the composite aerogel is lamellar. There are two reasons for this morphology. First, CMC molecular chains entangle with lamellar ES-LDHs to form these layered structures [37-39]. Second, the composite aerogel is prepared by the classic freeze-casting and then freeze-drying method, the resulting pores are the routes left by the ice crystals [40,41]. However, because of the relatively rigid molecule structure of CMC and intermolecular crosslinking, ice crystals can not grow freely in the gel network, and therefore make pore structure basically in disorder. In general, composite aerogels have porous structure in the microscale, which is the basis of high specific surface area.

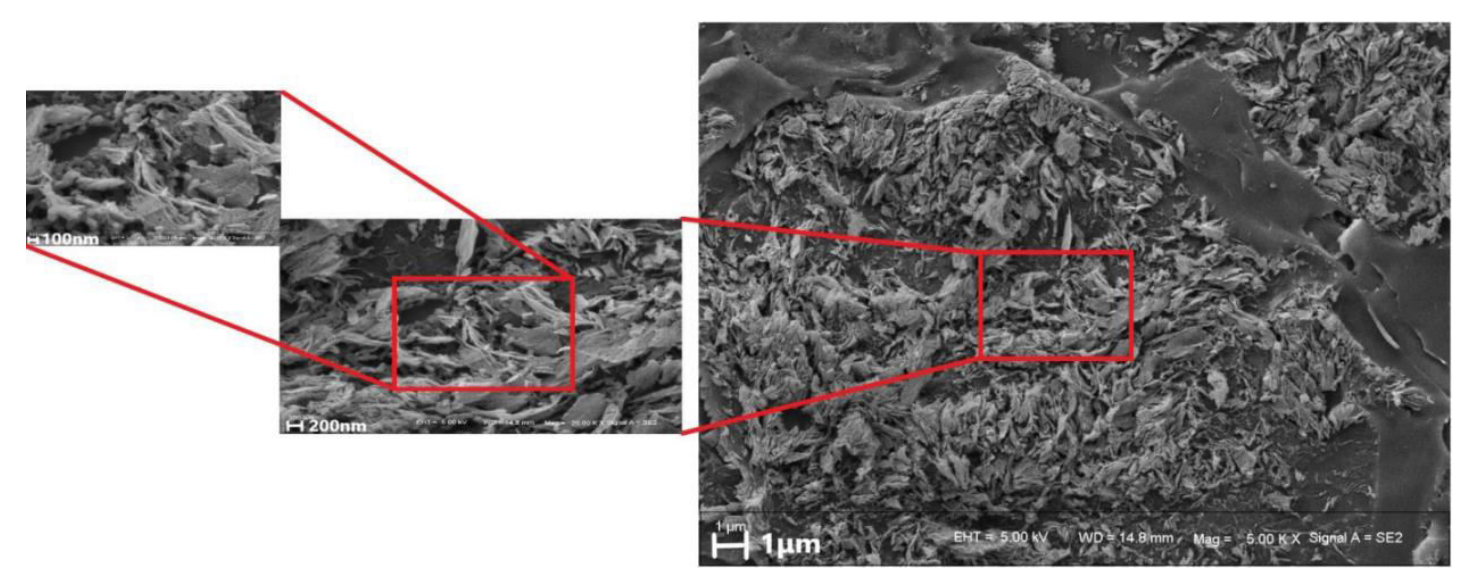

Figure 4. SEM photograph of ES-LDH@CMC-30 composite aerogel. 
The adsorption-desorption curves and corresponding pore size distribution of ES-LDH@CMC-30 composite aerogel are determined by BET method [42-44], and the results show in Figure 5. Based on the $\mathrm{N}_{2}$ adsorption-desorption curves, the adsorption isotherm hysteresis loop is complex $\mathrm{H} 3$ type. Due to the nano flake morphology of ES-LDH particles, the pore structure is slit, crack and wedge-shaped, and there is no saturated adsorption under high pressure. From the pore size distribution, it can be seen that the highest pore volume in the mesoporous range, indicating that the pore is mainly mesoporous. The specific surface area is deduced as $6.0854 \mathrm{~m}^{2} / \mathrm{g}$, which could be attributed to the randomly distributed ES-LDH lamellae in the CMC molecular network. The pore capacity of the aerogel is $0.012144 \mathrm{~cm}^{3} / \mathrm{g}$ and the average pore size is $7.9822 \mathrm{~nm}$ by the single point method.
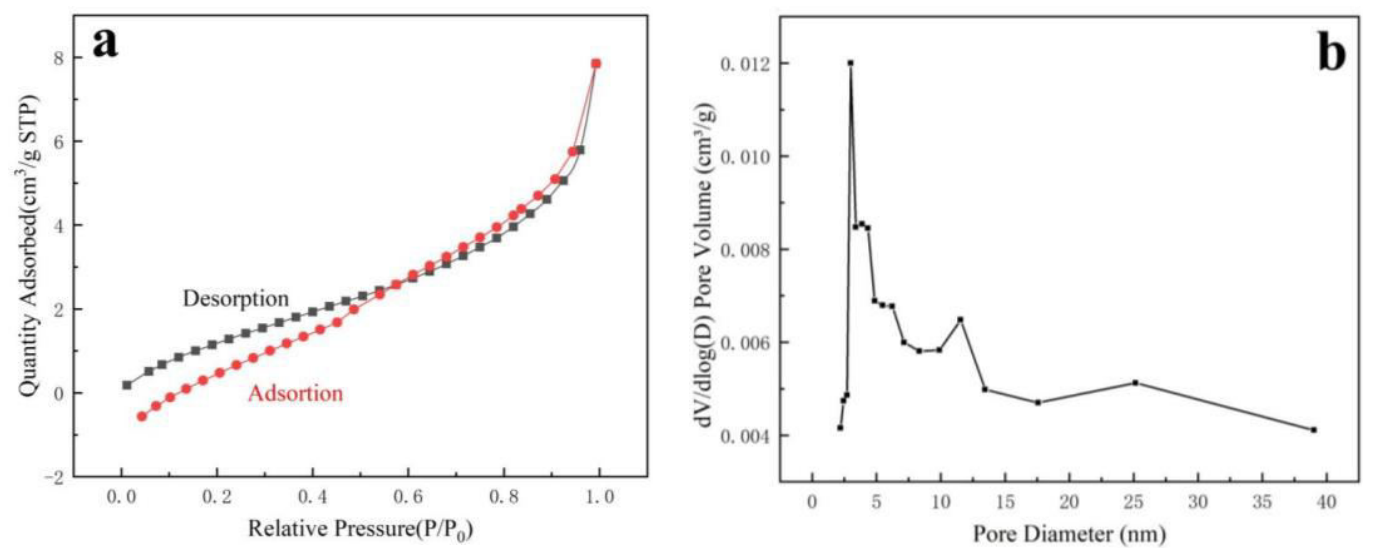

Figure 5. Adsorption-desorption curves (a) and corresponding pore size distribution (b) of ES-LDH@CMC-30 composite aerogel.

\subsection{Absorption behaviors of ES-LDH@CMC composite aerogels for Co(II)}

3.3.1 Adsorption capacity. To understand the absorption behavior of the material, the absorption capacities of kinds of LDHs@CMC composite aerogels for Co(II) are first recorded (Figure 6). The adsorption capacity of pristine MgFe-LDH@CMC composite aerogel to $\mathrm{Co}$ (II) is relatively low, which is ca. $22 \mathrm{mg} / \mathrm{g}$. As discussed above, the layer spacing of LDH is widened by SDBS intercalation, which also could form $\mathrm{Co}(\mathrm{DBS})_{2}$ compounds. However, this modification only slightly improves the adsorption capacity. In comparison, the adsorption of $\mathrm{Co}(\mathrm{II})$ by LDH with only EDTA@CMC composite aerogel increases to $30 \mathrm{mg} / \mathrm{g}$, which exhibits the significant role of complexation reaction. 
Finally, when SDBS and EDTA are used together as intercalators, i.e. ES-LDH@CMC aerogel, the adsorption capacity exceeds $40 \mathrm{mg} / \mathrm{g}$, which shows that the physical expansion and chemical complexation collaboratively work and remarkably promote the adsorption of $\mathrm{Co}(\mathrm{II})$.

For the ES-LDH@CMC aerogels, when the ES-LDH content in the aerogel is low, the increase of the adsorption capacity of the composite aerogels is due to the increase of CMC content and the corresponding adsorption sites of hydroxyl groups on the polymers. With high ES-LDH contents, the adsorption behavior of composite aerogels is closely related to the adsorption of ES-LDH, and the intercalation of EDTA and SDBS. When the content of ES-LDH is 30\%, CMC polymers and ES-LDH adsorption cooperate with each other, making the total adsorption amount reach the maximum.

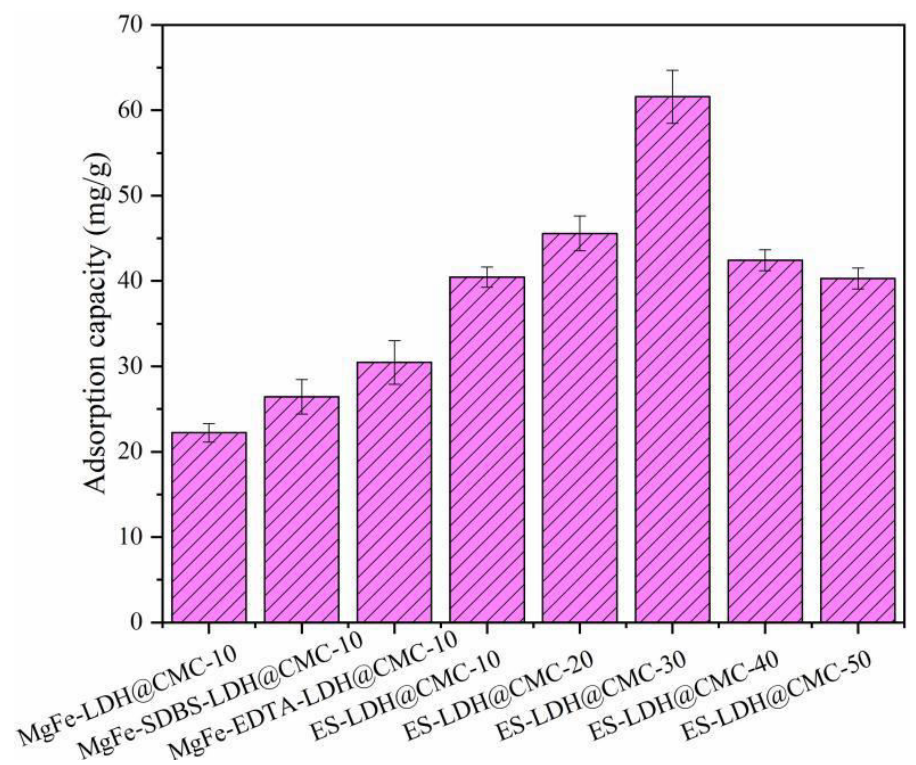

Figure 6. Absorption of composite aerogels for Co(II).

3.3.2 Absorption kinetics. To analyse the absorption behavior, the absorption kinetics of ES-LDH@CMC-30 for Co(II) are further fitted by pseudo first-order adsorption kinetic model (Figure 7A) and pseudo second-order adsorption kinetic model (Figure 7B). The correlation coefficients $\left(R^{2}\right)$ for the pseudo-second equation is close to 1 and obviously larger than that of pseudo first-order equation. The reason for relative inapplicability of the pseudo first-order model could be attributed to that the limited boundary layer controls the absorption process. The second-order reaction of absorption suggests the rate control process may be a chemical adsorption. In fact, the functional groups, such as hydroxyl, carboxyl and amine groups, could contribute to the chemisorption of $\mathrm{Co}(\mathrm{II})$ in the composite aerogel $[45,46]$. 
Based on the time-adsorption quantity data, intra-particle diffusion model (Figure 7C) and Elovich equation (Figure 7D) are also applied for consideration of mechanism. There are three stages for intra-particle diffusion: (1) external surface adsorption, (2) the gradual adsorption where intra-particle diffusion is rate-controlled and (3) intra-particle diffusion started to slow down because of decreasing concentration of adsorbate. Furthermore, the absorption rate could be controlled by the size of the adsorbate molecule, the concentration of the adsorbate, the affinity between adsorbate and adsorbent, the pore-size distribution in the adsorbent, the diffusion coefficient of the adsorbate and the degree of mixing [47]. In addition, because the clay-based ES-LDH particles could not completely uniformly distributed in the macromolecular network, and the surfaces of ES-LDHs have heterogeneous adsorption energy of $\mathrm{Co}(\mathrm{II})$, which fits the description of Elovich model [48]. All the deduced parameters of kinetic models are summarized in Table 2.
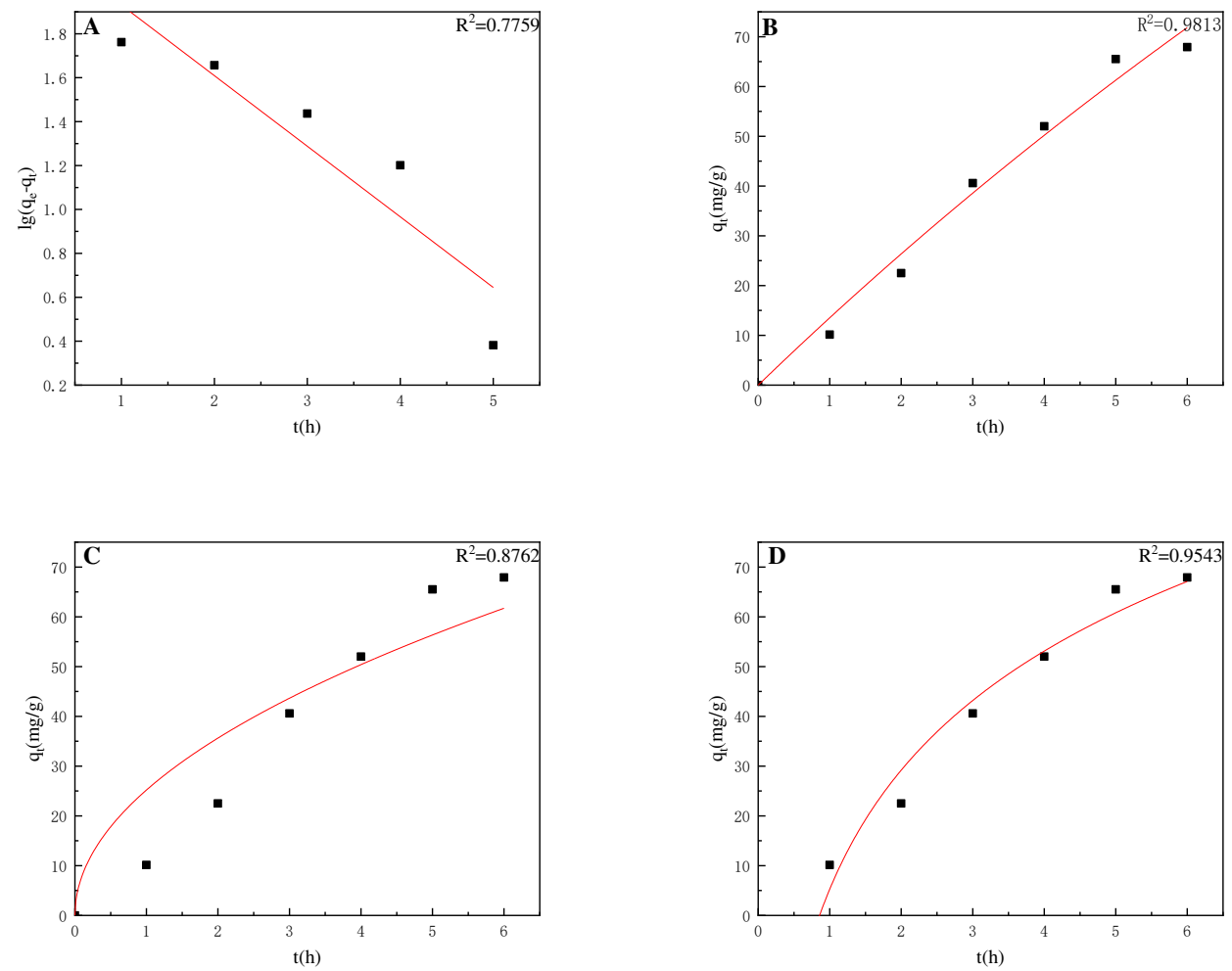

Figure 7. Plots of (A) pseudo first-order adsorption kinetic model, (B) pseudo second-order adsorption kinetic model, (C) particle diffusion model and (D) Elovich equation.

Table 2. Fitting parameters of the applied kinetic models 


\begin{tabular}{ccc}
\hline Pseudo first-order adsorption kinetic model & $k_{l}=0.6023$ & 0.7759 \\
Pseudo second-order adsorption kinetic model & $k_{2}=3.8501 \times 10^{-4}$ & 0.9813 \\
Particle diffusion model & $k=25.1976$ & 0.8762 \\
Elovich equation & $\alpha=40.2042, \beta=0.0290$ & 0.9543 \\
\hline
\end{tabular}

3.3.3 Adsorption isotherms. The adsorption isotherms of ES-LDH@CMC-30 for Co(II) are fitted by Langmuir, Freundlich, Temkin and D-R equations (Figure 8) and the relevant parameters are listed in Table 3. Based on these results, the absorption isotherm of ES-LDH@CMC-30 composite aerogel for $\mathrm{Co}$ (II) is more consistent with the descriptions of the first three equations, but less consistent with the description of D-R equation. The reason could be first attributed to the large specific surface area of the composite aerogel. Because of the insertion of SDBS into ES-LDH, the interlayer spacing of LDH expands, the specific surface area and physical adsorption capacity further increase. In addition, due to complexation function of EDTA, the composite aerogels contain a large number of $\mathrm{O}, \mathrm{N}$ groups, chemical adsorption ability of the materials are further enhanced. Through the chemisorption sites, there are both monolayer covered adsorption and heterogeneous multilayer adsorption inside the composites, which are in line with the description of Langmuir, Freundlich and Temkin models [46, 49, 50]. For the D-R model, although the correlation degree is the lowest, which is still greater than 0.9 , indicating that the behavior still has the characteristics of pore adsorption [51]. However, because of the relatively large pore size, the absorption behavior could not fully conform to the characteristics of D-R model.
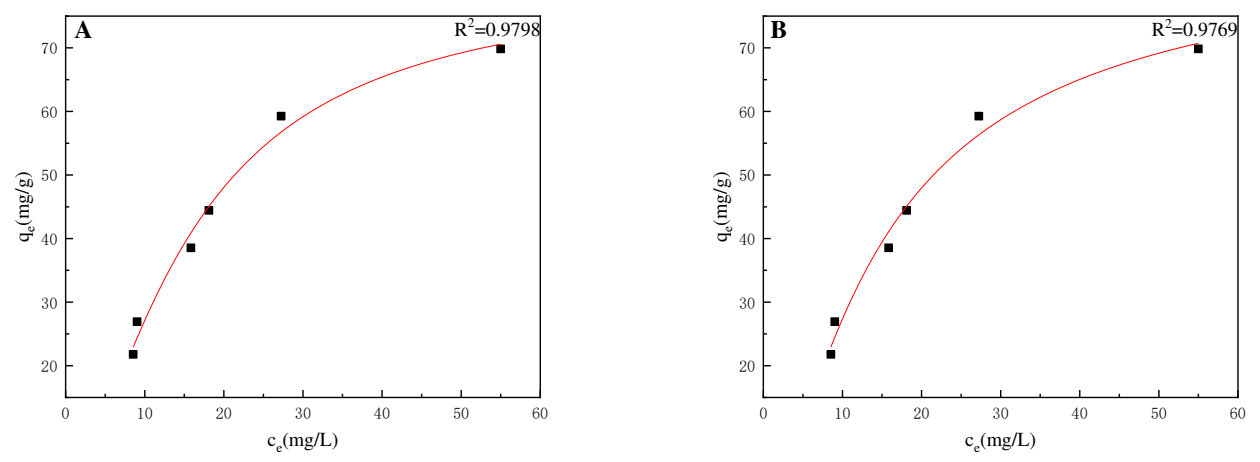

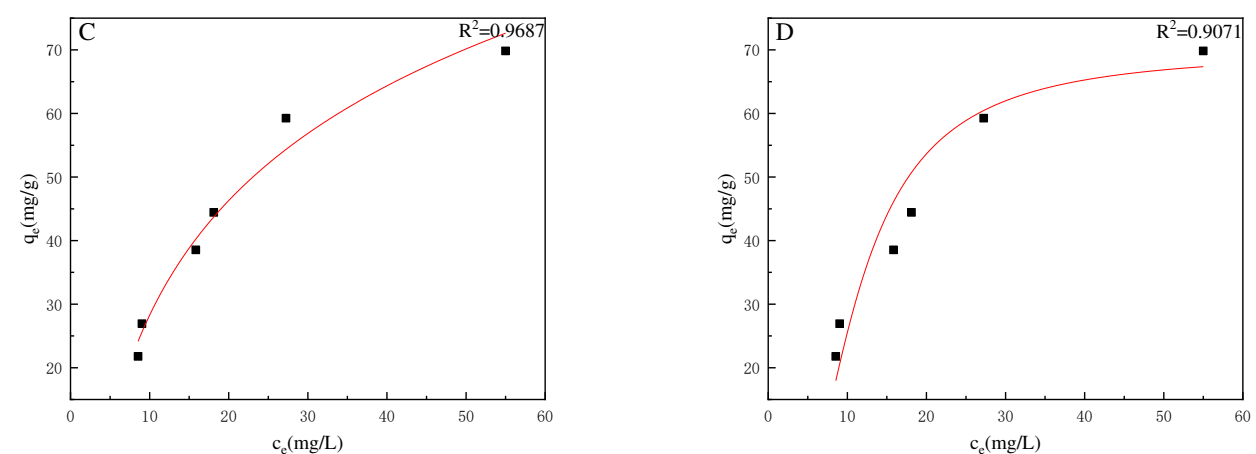

Figure 8. Plots of (A) Langmuir, (B) Freundlich, (C) Temkin and (D) D-R models.

Table 3. The models of adsorption isotherms and deduced parameters

\begin{tabular}{ccc}
\hline Model & Parameters & $\mathbf{R}^{\mathbf{2}}$ \\
\hline Langmuir & $q_{m}=80.62, k_{L}=0.0224$ & 0.9798 \\
Freundlich & $n=-1.7887, k_{F}=86.7316$ & 0.9769 \\
Temkin & $A_{T}=0, B_{T}=0.0107$ & 0.9687 \\
Dubinin-Radushkevich & $E=52.5, q_{m}=69.87$ & 0.9071 \\
\hline
\end{tabular}

\subsection{Absorption mechanism}

The adsorption of pristine $\mathrm{MgFe}-\mathrm{LDH}$ on $\mathrm{Co}(\mathrm{II})$ is based on its huge specific surface area and abundant oxygen-containing groups on the inner surface. Nevertheless, the adsorption capacity is not high. SDBS has a larger molecular volume. After exchanging with $\mathrm{CO}_{3}{ }^{2-}$ in the pristine $\mathrm{MgFe}-\mathrm{LDH}$, the ES-LDHs have significantly wider layer spacing, more smooth movement space for Co(II), and better complexation conditions of EDTA molecules with Co(II). In addition, SDBS molecule itself can also form complex compound of $\mathrm{Co}(\mathrm{DBS})_{2}$ with $\mathrm{Co}(\mathrm{II})$ to promote the adsorption of $\mathrm{Co}(\mathrm{II})$ [52]. As we see, this effect is not significant.

Furthermore, the adsorption behaviors of the composite aerogels can be further analyzed by combining adsorption kinetics and isotherms. The basic assumption of the Langmuir theory is that is all the adsorption sites have equal adsorbate affinity and the adsorption at one point does not affect the surrounding points. Therefore, uptake of adsorbate occurs on a homogenous surface in a monolayer without any inter-action between the adsorbates. Therefore, this model estimates the maximum adsorption capacity in a complete monolayer coverage. Among the four isotherms, the Langmuir model has the highest regression coefficient, suggesting generally uniform adsorption for $\operatorname{Co(II)}$ in the material. There are three main functional groups in the composite aerogel for $\mathrm{Co}(\mathrm{II})$ uptake. First, -OH groups from both LDHs and CMC, which could participate in the formation of the coordinate bonds 
[53]. Second, EDTA molecules could contribute more N, O-containing groups in the chelation process, which will result in higher adsorption capacity [54]. Third, SDBS molecules expand the interlayer spacing, as mentioned above. This absorption behavior indicates the efficiency and uniform distribution of these functional groups and molecules. The Freundlich adsorption isotherm and the Temkin adsorption isotherm are related to the concentration of the adsorbate and the adsorption potentials of the adsorbent for adsorbates, respectively. The relatively high regression coefficients imply that multilayer adsorption behavior exists. This behavior is reasonable because of intercalated modification by the rich functional molecules. The D-R model is to estimate the apparent free energy $(E)$ of adsorption. From the high value of the fitting curve, it verifies the strong chemisorption behavior of the composite aerogel [55].

\section{Conclusion}

In this study, SDBS and EDTA are intercalated in pristine MgFe-LDH to obtain a novel ES-LDH. Then, ES-LDH@CMC composite aerogel is fabricated. The intercalation of SDBS and EDTA in the LDH not only expands the interlayer spacing, but also increases the active adsorption groups, which significantly improves the adsorption capacity of the composite aerogel to Co(II) ions. This design solves the disadvantage of the difficulty in separation of LDH from water when it is used for adsorption. Simultaneously, the raw materials of this composite aerogel is easy to obtain, which will not add burden to the environment. The adsorption kinetics analysis show that the pseudo second-order model and Elovich model fit well with the adsorption processes, which reveals that the adsorption mainly depends on chemical complexation. And the adsorption isotherms fit well with the Langmuir, Freundlich and Temkin models. The reason could be attributed to the porous properties of aerogels and the lamellar structure of the $\mathrm{LDH}$, which results in a variety of adsorption behaviors inside the composite aerogels.

\section{Data availability statements}

All data generated or analysed during this study are included in this published article.

\section{Acknowledgement}

The author appreciate the financial support from the National Natural Science Foundation of China 
(Grants 51603130), the International Clean Energy Talent of China Scholarship Council and 'Young Scholars' support plan of Xihua University.

\section{Reference}

1. Awual, M.R., et al., $p H$ dependent $C u(I I)$ and $P d(I I)$ ions detection and removal from aqueous media by an efficient mesoporous adsorbent. Chemical Engineering Journal, 2014. 236: p. 100-109.

2. Juberg, D.R., C.F. Kleiman, and S.C. Kwon, Position paper of the American Council on Science and Health: lead and human health. Ecotoxicology and environmental safety, 1997. 38(3): p. 162-80.

3. Wang, J., et al., Selective removal of heavy metal ions in aqueous solutions by sulfide-selector intercalated layered double hydroxide adsorbent. Journal of Materials Science \& Technology, 2019. 35(9): p. 1809-1816.

4. Xiao, Y., et al., Functional covalent organic framework for exceptional $\mathrm{Fe} 2+, \mathrm{Co}(2+)$ and $\mathrm{Ni2}+$ removal: An upcycling strategy to achieve water decontamination and reutilization as smoke suppressant and flame retardant simultaneously. Chemical Engineering Journal, 2021. 421.

5. Jiang, C., et al., Adsorption performance of a polysaccharide composite hydrogel based on crosslinked glucan/chitosan for heavy metal ions. Composites Part B-Engineering, 2019. 169: p. $45-54$.

6. Li, S., et al., Graphene oxide based dopamine mussel-like cross-linked polyethylene imine nanocomposite coating with enhanced hexavalent uranium adsorption. Journal of Materials Chemistry A, 2019. 7(28): p. 16902-16911.

7. Chen, H., et al., Polydopamine modified cyclodextrin polymer as efficient adsorbent for removing cationic dyes and Cu2+. Journal of Hazardous Materials, 2020. 389.

8. Huang, X.Q., et al., Metal Sulfide Nanomaterials Based Adsorbents. Progress In Chemistry, 2017. 29(1): p. 83-92.

9. Liang, Q.W., et al., Fast and selective removal of Cr(VI) from aqueous solutions by a novel magnetic Cr(VI) ion-imprinted polymer. Journal of Molecular Liquids, 2017. 248: p. 767-774.

10. Huang, G., et al., Intercalation of thiacalix[4]arene anion via calcined/restored reaction into LDH and efficient heavy metal capture. Journal of Molecular Liquids, 2016. 220: p. 346-353.

11. Santos, R.M.M., et al., Thermal decomposition and recovery properties of ZnAl-CO3 layered double hydroxide for anionic dye adsorption: insight into the aggregative nucleation and growth mechanism of the LDH memory effect. Journal of Materials Chemistry A, 2017. 5(20): p. 9998-10009.

12. Palza, H., K. Delgado, and J. Govan, Novel magnetic CoFe2O4/layered double hydroxide nanocomposites for recoverable anionic adsorbents for water treatment. Applied Clay Science, 2019. 183.

13. Samuei, S., Z. Rezvani, and A.R. Amani-Ghadim, Comparative study of removal of reactive dye by LDHs: The effect of cation variety. Environmental Progress \& Sustainable Energy, 2017. 36(2): p. 372-381.

14. Razani, S. and A. Dadkhah Tehrani, Development of new organic-inorganic, hybrid 
bionanocomposite from cellulose nanowhisker and Mg/Al-CO3-LDHfor enhanced dye removal. Int J Biol Macromol, 2019. 133: p. 892-901.

15. Mallakpour, S. and M. Hatami, Biosafe organic diacid intercalated LDH/PVC nanocomposites versus pure $L D H$ and organic diacid intercalated LDH: Synthesis, characterization and removal behaviour of $\mathrm{Cd} 2+$ from aqueous test solution. Applied Clay Science, 2017. 149: p. 28-40.

16. Shi, M., et al., A novel heat-treated humic acid/MgAl-layered double hydroxide composite for efficient removal of cadmium: Fabrication, performance and mechanisms. Applied Clay Science, 2020. 187.

17. Sheng, G., et al., Enhanced sequestration of Cr(VI) by nanoscale zero-valent iron supported on layered double hydroxide by batch and XAFS study. Chemosphere, 2016. 148: p. 227-232.

18. Wang, F. and Z. Guo, Insitu growth of ZIF-8 on Co Al layered double hydroxide/carbon fiber composites for highly efficient absorptive removal of hexavalent chromium from aqueous solutions. Applied Clay Science, 2019. 175: p. 115-123.

19. Chen, H., et al., Preparation of MgAl-EDTA-LDH based electrospun nanofiber membrane and its adsorption properties of copper(II) from wastewater. Journal of Hazardous Materials, 2018. 345: p. 1-9.

20. Hu, B., et al., Macroscopic and spectroscopic insights into the mutual interaction of graphene oxide, $\mathrm{Cu}(\mathrm{II})$, and Mg/Al layered double hydroxides. Chemical Engineering Journal, 2017. 313: p. 527-534.

21. Yang, X.Y. and B. Al-Duri, Kinetic modeling of liquid-phase adsorption of reactive dyes on activated carbon. Journal of Colloid and Interface Science, 2005. 287(1): p. 25-34.

22. Ho, Y.S., Second-order kinetic model for the sorption of cadmium onto tree fern: A comparison of linear and non-linear methods. Water Research, 2006. 40(1): p. 119-125.

23. Weber, W.J. and J.C. Morris, Kinetics of Adsorption on Carbon from Solution. Journal of the Sanitary Engineering Division, 1963, Vol. 89, Issue 2, Pg. 31-60, 1963. 89(2): p. 31-60.

24. Ho, Y.S., W.T. Chiu, and C.C. Wang, Regression analysis for the sorption isotherms of basic dyes on sugarcane dust. Bioresource Technology, 2005. 96(11): p. 1285-1291.

25. Altın, O., H.O. Ozbelge, and T. Dogu, Use of general purpose adsorption isotherms for heavy metal-clay mineral interactions. Journal of Colloid and Interface Science, 1998. 198: p. 130-140.

26. Memon, J.R., et al., Banana peel: A green and economical sorbent for the selective removal of Cr(VI) from industrial wastewater. Colloids and Surfaces B-Biointerfaces, 2009. 70(2): p. 232-237.

27. Kumar, P.A., M. Ray, and S. Chakraborty, Adsorption behaviour of trivalent chromium on amine-based polymer aniline formaldehyde condensate. Chemical Engineering Journal, 2009. 149(1-3): p. 340-347.

28. Deng, L., et al., Fabrication of a novel NiFe2O4/Zn-Al layered double hydroxide intercalated with EDTA composite and its adsorption behavior for Cr(VI) from aqueous solution. Journal of Physics and Chemistry of Solids, 2017. 104: p. 79-90.

29. Zaghloul, A., et al., Removal and Comparative Adsorption of Anionic Dye on Various MgAl synthetic Clay. Biointerface Research In Applied Chemistry, 2021. 11(6): p. 14986-14997.

30. Zhang, D., et al., Effective removal of brilliant green from aqueous solution with magnetic Fe3O4@SDBS@LDHs composites. Transactions of Nonferrous Metals Society of China, 2017. 
27(12): p. 2673-2681.

31. Huang, D., et al., Cr(VI) removal from aqueous solution using biochar modified with Mg/Al-layered double hydroxide intercalated with ethylenediaminetetraacetic acid. Bioresource Technology, 2019. 276: p. 127-132.

32. Qiu, X. and W. Wang, Removal of borate by layered double hydroxides prepared through microwave-hydrothermal method. Journal of Water Process Engineering, 2017. 17: p. 271-276.

33. Rahman, S., et al., High capacity aqueous phosphate reclamation using Fe/Mg-layered double hydroxide $(L D H)$ dispersed on biochar. Journal of Colloid and Interface Science, 2021. 597: p. 182-195.

34. Fu, D., et al., Recovering heavy metals from electroplating wastewater and their conversion into Zn2Cr-layered double hydroxide $(\mathrm{LDH})$ for pyrophosphate removal from industrial wastewater. Chemosphere, 2021. 271.

35. Pavel, O.D., et al., Catalytic behavior of Li-Al-LDH prepared via mechanochemical and co-precipitation routes for cyanoethylation reaction. Catalysis Today, 2021. 366: p. 227-234.

36. Huang, Z., et al., Phosphotungstic acid intercalated Zn,Al-layered double hydroxides/ nanocellulose based $3 D$ lightweight foam thermal insulation materials. Materials Research Express, 2021. 8(2).

37. Huang, C., et al., Preparation of iron-based metal-organic framework @ cellulose aerogel by in situ growth method and its application to dye adsorption. Journal of Solid State Chemistry, 2021. 297.

38. $\mathrm{Xu}, \mathrm{J}$. , et al., The aminosilane functionalization of cellulose nanocrystal aerogel via vapor-phase reaction and its $\mathrm{CO} 2$ adsorption characteristics. Journal of Applied Polymer Science: p. 12.

39. Shahzadi, K., et al., Fire retardant cellulose aerogel with improved strength and hydrophobic surface by one-pot method. Journal of Applied Polymer Science, 2021. 138(16).

40. Liao, W., et al., On controlling aerogel microstructure by freeze casting. Composites Part B: Engineering, 2019. 173: p. 107036.

41. Scotti, K.L. and D.C. Dunand, Freeze casting - A review of processing, microstructure and properties via the open data repository, FreezeCasting.net. Progress In Materials Science, 2018. 94: p. 243-305.

42. Albadarin, A.B., et al., Activated lignin-chitosan extruded blends for efficient adsorption of methylene blue. Chemical Engineering Journal, 2017. 307: p. 264-272.

43. Naushad, M., et al., Nickel ferrite bearing nitrogen-doped mesoporous carbon as efficient adsorbent for the removal of highly toxic metal ion from aqueous medium. Chemical Engineering Journal, 2017. 330: p. 1351-1360.

44. Alqadami, A.A., et al., Novel Metal-Organic Framework (MOF) Based Composite Material for the Sequestration of U(VI) and Th(IV) Metal Ions from Aqueous Environment. ACS Applied Materials \& Interfaces, 2017. 9(41): p. 36026-36037.

45. Jing, Q.-X., et al., Behavior of ammonium adsorption by clay mineral halloysite. Transactions of Nonferrous Metals Society of China, 2017. 27(7): p. 1627-1635.

46. Li, J.-J., et al., Synthesis and Phosphorus Adsorption of Coal-Fly-Ash Magnetic Adsorbents. Chinese Journal of Inorganic Chemistry, 2018. 34(8): p. 1455-1462.

47. El-Kamash, A.M., A.A. Zaki, and M.A. El Geleel, Modeling batch kinetics and 
thermodynamics of zinc and cadmium ions removal from waste solutions using synthetic zeolite A. Journal of Hazardous Materials, 2005. 127(1-3): p. 211-220.

48. Chien, S.H. and W.R. Clayton, Application of Elovich equation to the kinetics of phosphate release and sorption on soils. Soil Science Society of America Journal, 1980. 44: p. 265-268.

49. Altun, T. and Y. Kar, Removal of Cr (VI) from aqueous solution by pyrolytic charcoals. New Carbon Materials, 2016. 31(5): p. 501-509.

50. Ruan, C.-P., K.-L. Ai, and L.-H. Lu, An Acid-resistant Magnetic Co/C Nanocomposite for Adsorption and Separation of Organic Contaminants from Water. Chinese Journal of Analytical Chemistry, 2016. 44(2): p. 224-230.

51. Akkaya, G. and A. Özer, Biosorption of Acid Red 274 (AR 274) on Dicranella varia: Determination of equilibrium and kinetic model parameters. Process Biochemistry, 2005. 40(11): p. 3559-3568.

52. Li, J., et al., Effect of surfactants on $\mathrm{Pb}(\mathrm{II})$ adsorption from aqueous solutions using oxidized multiwall carbon nanotubes. Chemical Engineering Journal, 2011. 166(2): p. 551-558.

53. Ma, J., et al., Fast adsorption of heavy metal ions by waste cotton fabrics based double network hydrogel and influencing factors insight. Journal of Hazardous Materials, 2018. 344: p. 1034-1042.

54. Godiya, C.B., et al., Carboxymethyl cellulose/polyacrylamide composite hydrogel for cascaded treatment/reuse of heavy metal ions in wastewater. Journal of Hazardous Materials, 2019. 364: p. 28-38.

55. Nasir, M.H., et al., Efficacy of modified distillation sludge of rose (Rosa centifolia) petals for lead(II) and zinc(II) removal from aqueous solutions. Journal of Hazardous Materials, 2007. 147(3): p. 1006-1014. 


\section{Supplementary Files}

This is a list of supplementary files associated with this preprint. Click to download.

- 3GraphicAbs.jpg 